\title{
Circulating Levels of Thrombospondin-1 and Thrombospondin-2 in Patients with Common Brain Tumors
}

\author{
Rahsan KEMERDERE ${ }^{1}$, Mehmet Yigit AKGUN ${ }^{1}$, Sureyya TOKLU ${ }^{1}$, Seckin AYDIN², Bagnu ORHAN ${ }^{3}$, \\ Berrin Bercik INAL ${ }^{3}$, Taha Sukru KORKMAZ ${ }^{1}$, Berkay AKTAS ${ }^{1}$, Tibet KACIRA ${ }^{4}$, Taner TANRIVERDI ${ }^{1}$ \\ ${ }^{1}$ Istanbul University-Cerrahpasa, Cerrahpasa Faculty of Medicine, Department of Neurosurgery, Istanbul, Turkey \\ ${ }^{2}$ University of Health Sciences, Okmeydani Training and Research Hospital, Department of Neurosurgery, Istanbul, Turkey \\ ${ }^{3}$ Istanbul Training and Research Hospital, Department of Clinical Biochemistry, Istanbul, Turkey \\ ${ }^{4}$ Sakarya University, Faculty of Medicine, Department of Neurosurgery, Istanbul, Turkey
}

Corresponding author: Rahsan KEMERDERE rahsank@istanbul.edu.tr

\section{ABSTRACT}

AIM: To measure serum levels of thrombospondin-1 (TSP-1) and thrombospondin-2 (TSP-2) in patients with common brain tumors, namely high-grade glioma (HGG), low-grade glioma (LGG), and meningioma.

MATERIAL and METHODS: For this prospective study, a total of 56 patients were operated on for supratentorial gliomas and meningiomas, and 18 healthy subjects were evaluated. Serum levels of angiostatic molecules were measured with enzyme-linked immunosorbent assay. The results of patients were compared with those of healthy subjects.

RESULTS: High serum levels of TSP-1 were seen in HGG, followed by LGG, meningioma groups, and controls. The only significant difference was found between HGGs and controls $(p=0.004)$. There was a trend to decrease from HGG to controls. High serum levels of TSP-2 were seen in controls, followed by meningioma, LGG, and HGG. None of the patient groups showed significant differences compared with controls. Among the patient groups, TSP-2 was significantly higher in the meningioma group than the HGG group ( $p=0.01)$. No correlation was found with any of the molecules and the clinical parameters, including the presence of peritumoral edema or seizure, the anterior-posterior diameter of the tumor, and, more importantly, the grade of glioma.

CONCLUSION: Our results indicate that TSP-2 might be more important than TSP-1 in preventing angiogenesis and a major angiostatic factor in glioma cells.

KEYWORDS: Angiogenesis, Brain tumors, Glioma, Meningioma, Thrombospondin

\section{INTRODUCTION}

$\mathrm{T}$ Thrombospondins (TSP) are matricellular proteins and comprises five members; TSP-1 through TSP-5. It has been demonstrated that TSP-1 and TSP-2 have similar structures that differ from other members of the family and are produced by several types of cells, including astrocytes (21). TSP-1, which was first isolated from platelets, is encoded by the TSP-1 gene (5). It has several important functions, such as the propagation of apoptosis, activation of transforming growth factor-beta (TGF- $\beta$ ), and immune regulation $(11,20)$. However, the main focus of interest in cancer research is the function of TSP-1 in the role of angiogenesis. It is a natural, anti-angiogenic, or angiostatic molecule (19). Similar to TSP1 , TSP-2 was highly expressed in developing blood vessels, suggesting its potential role in the regulation of angiogenesis. Thus, we currently know from cancer studies that both TSP1 and TSP-2 have important roles against angiogenesis (10).
Rahsan KEMERDERE (1) : 0000-0002-9947-3486 Mehmet Yigit AKGUN : : :0000-0003-1342-7663 Sureyya TOKLU (D) : 0000-0001-9396-8202 Seckin AYDIN (D): 0000-0003-1195-9084
Bagnu ORHAN (D) :0000-0003-1779-7784 Berrin Bercik INAL (D): 0000-0002-9098-4140 Taha Sukru KORKMAZ (10): 0000-0002-5678-257X Berkay AKTAS (1) : 0000-0003-4052-0695
Tibet KACIRA (D) : 0000-0003-4870-7550 Taner TANRIVERDI (10) : 0000-0002-1878-0696 
Global expression analysis from glioma cell lines revealed that the expression of TSP-1 is up-regulated in high-grade gliomas (HGG) and is associated with poor prognosis $(2,6,12)$. It has been proposed that high levels of TSP-1 in HGG are the result of the expression by glioma cells (15). Furthermore, TSP-1 disrupts the vasculature of growing tumors by inducing endothelial cell apoptosis. On the other hand, some findings revealed that high levels of TSP-1 in gliomas might be involved in the migration of glioma cells via interaction with its receptors, such as av $\beta 3$ and a3 $\beta 1$ integrins, thus propagating glioma growth (12). Others showed that the loss of tumor suppressors on chromosome 10 contributes to the malignant transformation from low-grade gliomas (LGG) to HGG, in part, by inhibiting the anti-angiogenetic action of TSP-1 (4). The lack of TSP-2 expression was associated with highergrade gliomas and increased vessel counts and density within glioma (7).

Angiogenesis and necrosis are important criteria for malignancy of a tumor. Cancer researchers have focused on the expression and functions of angiogenic factors and angiostatic agents to figure out the basic mechanism(s) behind tumor growth and malignant transformation. The data on the circulating levels of TSP-1 and TSP-2 in tumor patients is limited, and even unavailable for glioma patients except for one study that revealed the serum levels of TSP-1 and other angiogenic factors (16).

The aim of the present study is to present, for the first time, the serum levels of both TSP-1 and TSP-2 in patients with common brain tumors; HGG, LGG, and one of the most vascularized extra-axial brain tumors, meningioma (MNG). The results were compared with each group and healthy subjects.

\section{MATERIAL and METHODS}

\section{Study Population}

This prospective clinical study consisted of patients with histologically proven HGG, LGG, and meningioma (MNG), according to the World Health Organization Classification 2016 (9). After hospitalization, all patients were studied by magnetic resonance imaging (MRI), with complementary spectroscopy or perfusion studies when indicated. When the histopathological diagnosis was proven, patients who had HGG glioma [grade-III and grade-IV (GBM)] or high-grade MNG (grade-II and grade-III) were consulted for radiotherapy and/or chemotherapy. All patients were followed-up by neurosurgical and/or radiation/medical oncology departments at regular intervals. The control group was composed of healthy subjects working at our neurosurgery department and voluntarily provided venous blood samples.

The study was approved by the Local Ethics Committee. All patients and controls were informed about the aim of the study and gave written informed consent to participate in the study. All the procedures in this study comply with the Declaration of Helsinki.

\section{Radiological Assessment}

Cranial MRI with and without contrast agent was obtained from the patients. Only supratentorial tumors were included in this study. The presence of peritumoral edema was defined as a region of increased T2 signal intensity. Measurements of maximum anterior-posterior diameter (APD) were performed using contrasted images in cases of HGGs and MNGs. In LGGs, FLAIR sequences were used for the measurement of APD.

\section{Sample Collection}

Venous blood samples were obtained from controls and patients before surgery. Blood was collected in a BD vacutainer tube and centrifuged at $1500 \times \mathrm{g}$ for 30 minutes. The serum was then stored at $-80^{\circ} \mathrm{C}$ until the laboratory analysis.

\section{Angiogenesis Markers and Biochemical Determinations}

In this study, angiogenesis markers were included human thrombospondin-1 (TSP-1) and thrombospondin-2 (TSP-2). Serum levels of TSP-1 and TSP-2 (ng/mL) were assessed by the quantitative sandwich enzyme-linked immunosorbent assay kits (Elabscience Biotechnology Inc., USA) according to the manufacturer's instructions.

\section{Statistical Analysis}

Results are given as means \pm standard deviations for continuous variables. Group comparisons were made by the non-parametric Mann-Whitney $U$ test, and bivariate correlation analysis was performed by Pearson's correlation test. All statistical analyses were performed with SPSS computer software, version 20.0 for Windows (SPSS, Chicago, USA). Values of $p$ less than $0.05(p<0.05)$ were considered statistically significant.

\section{RESULTS}

\section{Demographic and Clinical Characteristics of Study Subjects}

This study included a total of 56 patients and 18 healthy controls. The surgery was performed between October 2018 and March 2019, and all tumors were in the supratentorial area. In this study, patients were grouped into three groups: HGG group (19 patients), LGG group (17 patients), and MNG group (20 patients). The mean age of controls was $33.3 \pm 6.9$ years; $44 \%$ were female. The mean ages of HGG and MNG groups were higher than those of the controls, and the differences were significant $(p=0.00001)$. However, no difference was found between the LGG group and controls regarding the mean age. There were no statistically significant differences among the patient groups $(p>0.05)$. Seizure was found commonly in LGG patients, and peritumoral edema was commonly found in HGG patients. The majority of histopathological diagnosis was grade-IV (GBM) in HGG and grade-I meningioma in MNG groups. In LGG, all patients were diagnosed with grade-II diffuse astrocytoma. The demographic and clinical characteristics are summarized in Table I.

\section{Circulating Levels of TSP-1 and TSP-2}

In this study, serum levels of TSP-1 and TSP-2 were measured in patients with HGG, LGG, and MNG and controls. The mean 
levels of the parameters in each group are summarized in Table II. Overall, serum levels of each molecule showed opposite directions. However, TSP-1 levels demonstrated a decline from HGG to controls, whereas TSP-2 showed an incline from the HGG group to the control group.

Comparisons between the controls and each patient group regarding TSP-1 showed that the only significant difference was between patients with HGG and controls $(p=0.004)$. Patients with $L G G$ and controls $(p=0.08)$, together with $M N G$ and controls $(p=0.15)$ showed non-significant differences (Figure 1A). Regarding TSP-2, none of the patient groups showed significant difference compared with controls $(p=0.23$, 0.5 , and 0.19 for HGG, LGG, and MNG, respectively) (Figure 1B).

Comparisons among patient groups showed that no significant differences were found between HGG and LGG $(p=0.26)$, $H G G$ and $M N G(p=0.19), L G G$ and $M N G(p=0.44)$ (Figure $1 A)$. The mean serum levels of TSP-2 were significantly higher

Table I: Clinical Characteristics of Patients and Controls

\begin{tabular}{|c|c|c|c|c|}
\hline${ }^{*}$ Parameters & HGG $(n=19)$ & LGG $(n=17)$ & MNG $(n=20)$ & Controls $(n=18)$ \\
\hline Mean age (years) & $53.4 \pm 18.03$ & $41.6 \pm 14.1$ & $53.7 \pm 16.1$ & $33.3 \pm 6.9$ \\
\hline Gender (female/male) & $7 / 12$ & $9 / 8$ & $14 / 6$ & $8 / 10$ \\
\hline Seizure (yes/no) & $7 / 12$ & $10 / 7$ & $6 / 14$ & \\
\hline Lateralization (right/left) & $8 / 11$ & $5 / 12$ & $12 / 8$ & \\
\hline Edema (yes/no) & $9 / 10$ & $5 / 12$ & $6 / 14$ & \\
\hline Diameter (mm) & $46.6 \pm 14.5$ & $46.1 \pm 17.07$ & $47.7 \pm 20.5$ & \\
\hline Glioma grade (II/III/IV) & $-/ 3 / 16$ & $17 /-/-$ & & \\
\hline MNG grade (I/II/III) & & & $16 / 3 / 1$ & \\
\hline
\end{tabular}

HGG: High-grade glioma, LGG: Low-grade glioma, MNG: Meningioma. *Values are given as mean \pm standard deviation where appropriate.

Table II: Mean ( \pm Standard Deviation) Serum Levels of Human Thrombospondin-1 (TSP-1) and Thrombospondin-2 (TSP-2) in Patients and Controls

\begin{tabular}{lcccc}
\hline${ }^{*}$ Parameters & HGG $(\mathbf{n}=\mathbf{1 9})$ & LGG $(\mathbf{n}=\mathbf{1 7})$ & MNG $(\mathbf{n}=\mathbf{2 0})$ & Controls $(\mathbf{n}=\mathbf{1 8})$ \\
\hline TSP-1 & $25140.22 \pm 7503$ & $23692.17 \pm 11259$ & $21582.67 \pm 4688$ & $19778.16 \pm 5269$ \\
\hline TSP-2 & $20.17 \pm 10.2$ & $24.90 \pm 9.2$ & $27.05 \pm 9.4$ & $32.25 \pm 25.3$ \\
\hline
\end{tabular}

HGG: High-grade glioma, LGG: Low-grade glioma, MNG: Meningioma. *Values are given as "ng/mL”.

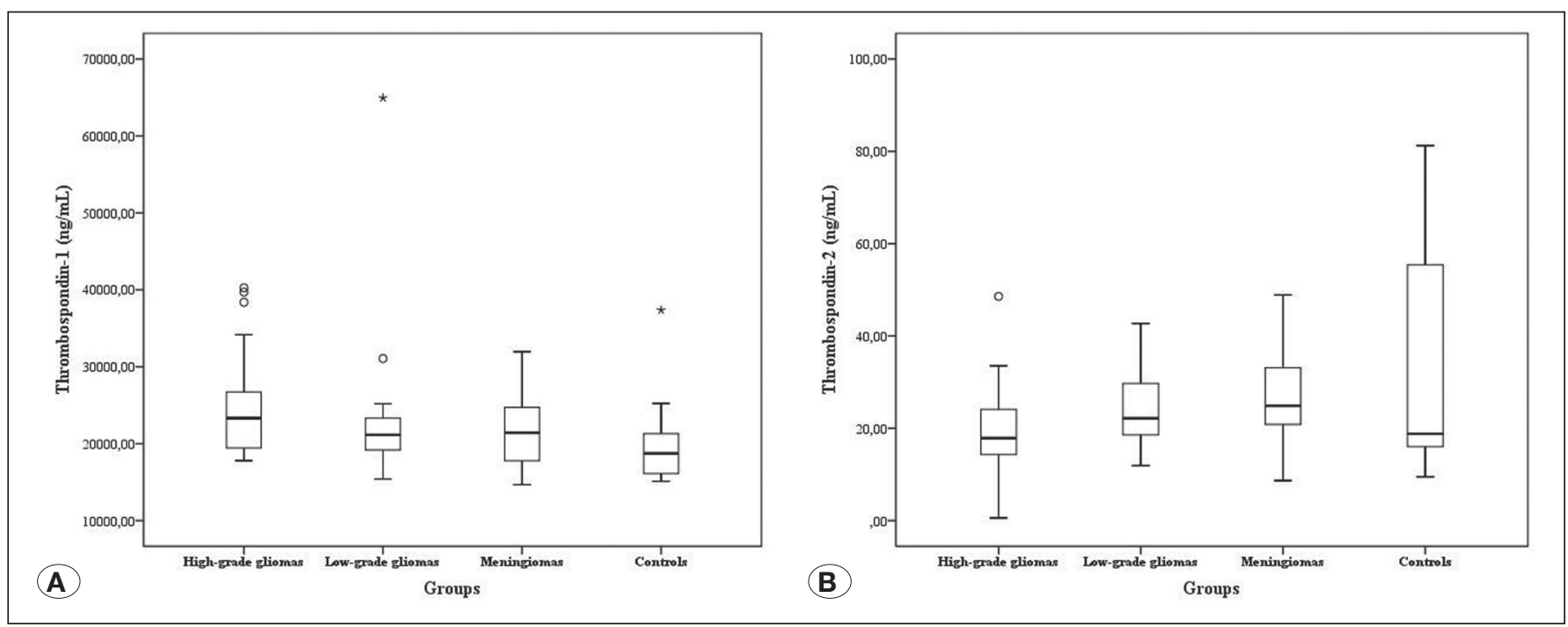

Figure 1: Summary of serum levels of TSP-1 (A) and TSP-2 (B) in patients and controls before surgery. The horizontal line inside the box is the mean, and the box represents the lower and upper quartiles. 
in the MNG group than the HGG group $(p=0.01)$. However, the differences were not significant between HGG and LGG $(p=0.13)$, and between $L G G$ and MNG $(p=0.41)$ with respect to TSP-2 (Figure 1B).

\section{Correlation Analysis}

No statistical associations were found between marker levels and clinical parameters, including the presence of seizure or peritumoral edema, APD of tumors, and the grade of glioma (p>0.05).

\section{DISCUSSION}

Histopathological diagnosis of malignancy, or becoming high-grade for a tumor, requires high mitotic activity, diffuse invasion to the surrounding, extensive angiogenesis, and necrosis (9). The progressive growth of a tumor depends on angiogenesis. In healthy cells, there is a precise balance between angiogenic and angiostatic (anti-angiogenic) mechanisms. It is clear that an angiogenic switch in highgrade tumors occurs that allows the secretion of high levels of inducers and low levels of molecules against angiogenesis (1). Thrombospondins are a group of five extracellular matrix molecules (TSP-1 to TSP-5) that involve several cellular processes, including the modulation of immune responses and vascularization $(5,11,19,20)$. TSP- 1 and TSP-2 have been demonstrated to have inhibitory properties on angiogenesis in various cancer cell lines, including gliomas $(2,4,6,7,12,15,22)$. The current literature suggested that augmentation of TSP-1 and/or inhibiting TSP-2 in HGG may be beneficial and should be involved in treatment regimens $(2,7)$. Thus, recent clinical research has focused on thrombospondins to see whether the angiogenic switch can be stopped by augmenting, particularly TSP-1 and TSP-2, in patients with HGG. However, there has been no consensus, and the results published so far are conflicting. The present study is the first to measure the serum levels of TSP-1 and TSP-2 concurrently in patients with common brain tumors and compare them with serum from healthy controls. We should emphasize that our findings are in line with some reports $(6,7,12)$, but are in opposition to others $(4,13,18)$. Namely, Kazuno et al. (7) demonstrated in 37 glioma patients that expression of the TSP-2 gene was significantly correlated with a lower grade than glioma lacking the TSP-2 gene. However, TSP-1 gene expression was not correlated with any grade of glioma. They presumed that TSP-2 gene expression might be an important factor for angiogenesis in human gliomas. Kawataki et al. investigated the expression of TSP-1 in a panel of malignant glioma cell lines and expression of TSP-1 in LGG and HGG tissues by immunohistochemistry (6). The majority of grade-IV gliomas showed strong immunostaining for TSP-1. TSP-1 was also localized in proliferated vascular cells within GBM. However, all anaplastic gliomas (grade-III) showed moderate staining for TSP-1. Interestingly, normal brain tissues far from glioma cells showed no or very weakly staining for TSP-1. They concluded that the expression of TSP-1 was correlated with the malignancy of glioma. They further speculated that increased angiogenesis, despite TSP-1 overexpression in GBM, might be because angiogenic factors, such as VEGF and basic
FGF, dominated TSP-1. Naganuma et al. (12) showed that malignant glioma cell lines secreted large amounts of TSP-1 and its receptors, integrins, and syndecan-1 compared with non-glioma malignant tumors. Only one study reported serum levels of specific angiogenesis markers, including TSP-1 in 47 GBM patients. They demonstrated a high serum level of TSP-1 in patients compared with healthy controls, but the difference was not significant (16). Most of our findings are in line with the above-mentioned studies. Serum levels of TSP-1 were higher in patients with HGG, LGG, and MNG than controls, and significantly higher levels were found only in patients with HGG. Regarding TSP-2, a significantly higher serum level of TSP-2 was found in HGG compared with MNG. TSP-1 and TSP-2 showed opposite directions. TSP-1 decreased from HGG (to LGG to MNG) to controls, whereas TSP-2 increased from HGG to controls. These unexpected opposite trends supported Kazuno et al. (7) and suggested that TSP-2 might be more important than TSP-1 in angiogenesis in HGGs. Overexpression of TSP-2 in LGG, MNG, and healthy controls proposed that TSP-2 may prevent the angiogenic switch in the normal brain or aforementioned pathological conditions. High levels of TSP-1 and low levels of TSP-2 may be related to the change in glioma cells to a malignant phenotype. In contrast to the current literature, we did not find a significant correlation between the serum levels of TSP-1/TSP-2 with the presence of seizure, edema, size of the tumor, and, more importantly, with the grade of glioma $(2,6,7)$.

The current literature shows that TSP- 1 activates latent TGF- $\beta$ in malignant glioma $(3,8,14,17)$. Active TGF- $\beta$ has strong immunosuppressive functions against tumor cells and inhibits cytokines, which are key players in immunological reactions. Thus, based on our results, we can speculate that secretion of high levels of TSP-1 and low levels of TSP-2 may decrease the immunological reactions around malignant gliomas and allow propagation of tumor growth. Some studies propose that TSP-1 inhibition may be therapeutically important to reinforce the current glioma treatment $(2,7)$. We also have to emphasize that results from the recent literature, including the present study, should be evaluated carefully because of diverse samples and different techniques used and the very limited number of patients included.

\section{CONCLUSION}

Taken together, our results indicate that TSP-2 might be more important than TSP-1 in preventing angiogenesis. TSP-2 is a major angiostatic factor in glioma cells. Both molecules can be detected in HGGs and LGGs, benign brain tumors such as meningioma, and even in the serum of healthy people.

\section{- REFERENCES}

1. Bouck N, Stellmach V, Hsu S: How tumors become angiogenic. Adv Cancer Res 69:135-174, 2006

2. Daubon T, Léon $C$, Clarke $K$, Andrique L, Salabert L, Darbo E, Pineau R, Guérit S, Maitre M, Dedieu S, Jeanne A, Bailly $\mathrm{S}$, Feige JJ, Miletic H, Rossi M, Bello L, Falciani F, Bjerkvig R, Bikfalvi A: Deciphering the complex role of thrombospondin-1 in glioblastoma development. Nat Commun 10:1146-1160, 2019 
3. Espevik T, Figari IS, Shalaby MR, Lackides GA, Lewis GD, Shepard HM, Palladino MA Jr: Inhibition of cytokine production by cyclosporin A and transforming growth factor B. J Exp Med 166:571-576, 1987

4. Hsu SC, Volpert OV, Steck PA, Mikkelsen T, Polverini PJ, Rao S, Chou P, Bouck NP: Inhibition of angiogenesis in human glioblastoma by chromosome 10 induction of thrombospondin-1.Cancer Res 56:5684-5691, 1995

5. Jaffe $E$, Bornstein $P$, Disteche $C M$ : Mapping of the thrombospondin gene to human chromosome 15 and Mouse chromosome 2 by in situ hybridization. Genomics 7:123-126, 1990

6. Kawataki T, Naganuma H, Sasaki A, Yoshikawa H, Tasaka K, Nukui $\mathrm{H}$ : Correlation of thrombospondin-1 and transforming growth factor- $\beta$ expression with malignancy of glioma. Neuropathol 20:161-169, 2000

7. Kazuno M, Tokunaga T, Oshika Y, Tanaka Y, Tsugane R, Kijima $\mathrm{H}$, Yamazaki H, Ueyama Y, Nakamura M: Thrombospondin-2 (TSP2) expression is inversely correlated with vascularity in glioma. Eur J Cancer 35:502-506, 1999

8. Kehrl JH, Wakefield LM, Roberts AB, Jakowlew S, AlvarezMon M, Derynck R, Sporn MB, Fauci AS: Production of transforming growth factor $\beta$ by human $T$ lymphocytes and its potential role in the regulation of T cell growth. J Exp Med 163:1037-1050, 1986

9. Kleihues P, Burger PC, Aldape KD, Brat DJ, Biernat W, Bigner DD: Glioblastoma. In: Louis DN, Ohgaki H, Wiestler OD, Cavenee WK et al. (eds), WHO classification of tumors of the Central Nervous System $4^{\text {th }}$ ed, Lyon: IARC, 2007: 33-49

10. Krady MM, Zeng J, Yu J, MacLauchlan S, Skokos EA, Tian W, Bornstein P, Sessa WC, Kyriakides TR: Thrombospondin-2 modulates extracellular matrix remodeling during physiological angiogenesis. Am J Pathol 173:879-891, 2008

11. Ma Y, Qu B, Xia X, Yang L, Kuang Y, Yang T, Cheng J, Sun $\mathrm{H}$, Fan K, Gu J: Glioma-derived thrombospondin-1 modulates cd14+ cell tolerogenic properties. Cancer Invest 33:152-157, 2015

12. Naganuma $H$, Satoh $E$, Asahara $T$, Amagasaki $K$, Watanabe A, Satoh H, Kuroda K, Zhang L, Nukui H: Quantification of thrombospondin-1 secretion and expression of $\operatorname{av} \beta 3$ and a3 $\beta$ integrins and syndecan-1 as cell-surface receptors for thrombospondin-1 in malignant glioma cells. J Neurooncol $70: 309-317,2004$
13. Pijuan-Thompson V, Grammer JR, Stewart J, Silverstein RL, Pearce SF, Tuszynski GP, Murphy-Ullrich JE, Gladson CL: Retinoic acid alters the mechanism of attachment of malignant astrocytoma and neuroblastoma cells to thrombospondin-1. Exp Cell Res 249:86-101, 1999

14. Ranges GE, Figari IS, Espevik T, Palladino MA Jr: Inhibition of cytotoxic $T$ cell development by transforming growth factor $\beta$ and reversal by recombinant tumor necrosis factor aa. J Exp Med 166:991-998, 1987

15. Reiher FK, Volpert OV, Jimenez B, Crawford SE, Dinney CP, Henkin J, Haviv F, Bouck NP, Campbell SC: Inhibition of tumor growth by systemic treatment with thrombospondin-1 peptide mimetics. Int J Cancer 98:682-689, 2002

16. Reynés G, Vila V, Martín M, Parada A, Fleitas T, Reganon E, Martínez-Sales V: Circulating markers of angiogenesis, inflammation, and coagulation in patients with glioblastoma. J Neurooncol 102:45-41, 2011

17. Schultz-Cherry S, Lawler J, Murphy-Ullrich JE: The type 1 repeats of thrombospondin 1 activate latent transforming growth factor- $\beta$. J Biol Chem 269:26783-26788, 1994

18. Tenan BM, Fulci G, Albertoni M, Diserens AC, Hamou MF, El Atifi-Borel M, Feige JJ, Pepper MS, Van Meir EG: Thrombospondin-1 is downregulated by anoxia and suppresses tumorigenicity of human glioblastoma cells. J Exp Med 191:1789-1798, 2000

19. Tolsma SS, Volpert OV, Good DJ, Frazier WA, Polverini PJ, Bouck N: Peptides derived from two separate domains of the matrix protein thrombospondin-1 have anti-angiogenic activity. J Cell Biol 122:497-511, 1993

20. Tuszynski GP, Nicosia RF: The role of thrombospondin-1 in tumor progression and angiogenesis. Bioessays 18:71-76, 1996

21. Wolf FW, Eddy RL, Shows TB, Dixit VM: Structure and chromosomal localization of the human thrombospondin gene. Genomics 6:685-691, 1990

22. Zabrenetzky V, Harris CC, Steeg PS, Roberts DD: Expression of the extracellular matrix molecule thrombospondin inversely correlates with malignant progression in melanoma, lung, and breast carcinoma cell lines. Int J Cancer 59:191-195, 1994 